\title{
miR-342-5p as a Potential Regulator of HER2 Breast Cancer Cell Growth
}

\section{Lindholm, Evita}

2019

Lindholm , E, Leivonen, S-K, Undlien, E, Nebdal, D, Git , A, Caldas , C, Borresen-Dale , A-L , Sahlberg , K \& Kleivi , K 2019 , ' miR-342-5p as a Potential Regulator of HER2 Breast Cancer Cell Growth ' , MicroRNA , vol. 8 , no. 2 , pp. 155-165 . https://doi.org/10.2174/221153660866618120612492

http://hdl.handle.net/10138/324810

https://doi.org/10.2174/2211536608666181206124922

acceptedVersion

Downloaded from Helda, University of Helsinki institutional repository.

This is an electronic reprint of the original article.

This reprint may differ from the original in pagination and typographic detail.

Please cite the original version. 


\section{miR-342-5p as a Potential Regulator of HER2 Breast Cancer Cell Growth}

Evita Lindholm ${ }^{1,}{ }^{*}$, Suvi-Katri Leivonen ${ }^{2}$, Eldri Undlien ${ }^{1}$, Daniel Nebdal ${ }^{1}$, Anna Git $^{3}$, Carlos Caldas ${ }^{3}$, Anne-Lise Børresen-Dale ${ }^{1}$ and Kristine Kleivi Sahlberg ${ }^{1,4}$

${ }^{1}$ Department of Cancer Genetics, Institute for Cancer Research, Oslo University Hospital, The Norwegian Radium Hospital, Oslo, Norway; ${ }^{2}$ Research Program Unit, Medical Faculty, University of Helsinki, Helsinki, Finland; ${ }^{3}$ Cancer Research UK Cambridge Institute, Li Ka Shing Centre, University of Cambridge, Cambridge, United Kingdom; ${ }^{4}$ Department of Research, Vestre Viken Hospital Trust, Drammen, Norway

Abstract: Background: HER2 positive breast cancers (BC) have aggressive behavior and poor prognosis. Previously, we have identified miR-342-5p as an upstream regulator of HER2 signaling, as well as inhibitor of HER2 positive BC cell line growth.

Objective: Here, we aimed to further investigate the molecular mechanisms behind miR-342-5pinduced HER2 pathway deregulation. Two HER2 amplified breast cancer cell lines were transiently transfected with miR-342-5p mimic or negative control, and gene expression was analyzed by Agilent microarrays.

Method: Three clinical datasets with BC patients were used to identify correlations between candidate genes and miR-342-5p, and associations with survival. Pathway analyses of all deregulated genes revealed a significant suppression of the HER2 downstream pathways ERK/MAPK and SAPK/JNK, whereas the miR-342-5p predicted target genes were enriched for pathways associated with cell motility.

Results: Biological functions linked to mitochondrial stability were ranked among the top toxicological functions in both gene lists. Among the most deregulated genes, Cytochrome B5 Reductase 3 (CYB5R3) and Rap Guanine Nucleotide Exchange Factor 6 (RAPGEF6) significantly anticorrelated and correlated, respectively, with miR-342-5p in all three clinical BC datasets. Low CYB5R3 levels and high RAPGEF6 levels were significantly associated with survival, although this was not directly associated with HER2 expression.

Conclusion: Our data suggest that miR-342-5p overexpression in HER2 positive BC cell lines elicits broad effects on HER2 downstream signaling, cell motility and mitochondrial stability. Together these effects may render cells less proliferative and more sensitive to cellular stress.

Keywords: CYB5R3, HER2 signaling, miR-342-5p, RAPGEF6. 


\section{INTRODUCTION}

In recent years, microRNAs (miRNAs) have revealed a wealth of new insights into the intrinsic regulation of transcription and translation of protein-coding RNAs. MicroRNAs are small, non-coding RNA molecules regulating gene-expression post-transcriptionally, most often through

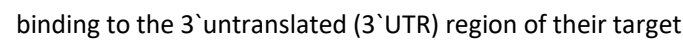
mRNAs. This causes translational repression or mRNA destabilization [1]. Over 2500 different miRNAs have been described in the human genome (http://microrna.sanger.ac.uk), and they have been identified as regulators of many biological processes, including proliferation, differentiation and cell death in both normal and cancer cells [2-4]. Furthermore, the ability of one miRNA to target hundreds of mRNAs [5] highlights their unique capability to modulate an immense and complex regulatory network of gene expression. The fact that miRNAs can work as oncogenes or tumor suppressors, depending on the cellular context in which they are expressed, has opened the potential use of miRNA mimics or antagonists as therapeutics [6]. The human epidermal growth factor receptor 2 (HER2), also known as ErbB2, is found overexpressed in about $15-20 \%$ of invasive breast cancers, and is associated with poor disease-free survival [7, 8]. HER2 overexpression leads to constitutive activation of downstream signaling, such as AKT and MAPK pathways, resulting in increased cell growth, survival and migration. In our previous study, we identified several miRNAs regulating cell growth and apoptosis, HER2 protein levels and inhibition of AKT and ERK1/2 downstream signaling mediators in HER2 positive cell lines. miR-342-5p was one of the most potent down-regulator of HER2 protein and downstream signaling, whereas no change in cell growth or apoptosis was seen in the HER2 negative, MCF7, cell line [9]. The expression of miR-342-5p was also found to be significantly higher in HER2 negative breast cancer patients compared to the HER2 positive patients, which may imply a potential role of this miRNA as a therapeutic strategy in the management of HER2 positive breast cancer. However, although high expression of miR-342-5p was significantly associated with a better overall survival in two cohorts of breast cancer patients [9], no specific link to the HER2 positive subset of breast cancer has been described. Furthermore, no direct binding between the 3 'UTR region of HER2 and miR-342$5 p$ was identified. Based on our previous findings we hypothesize that miR-342-5p exerts its tumor inhibiting effects through mechanisms that are either separate or indirectly linked to the HER2 receptor. To further unravel the mechanisms by which miR-342-5p modulates the HER2 signaling pathways and cell growth, we thus performed mRNA expression analyses after miR-342-5p overexpression in two HER2-amplified breast cancer cell lines, KPL-4 and JIMT-1. By combining the gene expression data with miRNA target predictions, we identified Cytochrome B5 Reductase 3 (CYB5R3) and Rap Guanine Nucleotide Exchange Factor 6 (RAPGEF6) as potential contributors to the tumor-inhibiting effects of miR-342-5p. The results presented here are important for the understanding of the molecular mechanisms underlying the role of miR-342-5p in HER2 positive breast cancer.

\section{MATERIALS AND METHODS}

\subsection{Cell Lines}

Two human HER2 positive, trastuzumab non-responsive breast cancer cell lines, KPL-4 and JIMT-1, were used to identify potential targets for miR-342-5p. KPL-4 cells were a kind gift from Prof. Junichi Kurebayashi (Kawasaki Medical School, Japan) [10], and they were cultured in DMEM (4.5 g/I glucose; Sigma-Aldrich, St. Louis, MO) supplemented with $10 \%$ fetal bovine serum (FBS), 2 mM l-glutamine and 1\% penicillin/streptomycin. JIMT-1 cells were obtained from the German Collection of Microorganisms and Cell Cultures (DSMZ, Leibniz, Germany), and were cultured in 1:1 Ham's F-12/DMEM (4.5 g/l glucose) supplemented with 10\% FBS, $10 \mu \mathrm{g} / \mathrm{ml}$ insulin, $2 \mathrm{mM}$ l-glutamine and $1 \%$ penicillin/ streptomycin.

\subsection{Transfections and RNA Isolation}

The cells were transiently transfected with $20 \mathrm{nM}$ precursor 
for miR-342-5p or negative control miRNA (Ambion

Inc., Austin, TX, USA) using SilentFect (Bio-Rad Laboratories,

Hercules, CA, USA) as described previously [9]. The

transfected cells were incubated for 24 hours, and total RNA

was extracted directly from each well using MiRVana ${ }^{\mathrm{TM}}$

Total RNAIsolation kit (Ambion Inc.) according to the manufacturer

s protocol. The transfections were done in three

separate biological replicates.

\section{EXPRESSION ARRAYS}

mRNA expression was measured using SurePrint G3

Human GE 8x60K one-color microarrays (Agilent Technologies)

according to the manufacturer's protocol (One-Color

Microarray-Based Gene Expression Analysis, Low Input

Quick Amp Labeling, v.6.5, May 2010). For each sample,

100 ng of RNA was amplified and hybridized on the array,

which includes 42,405 unique 60 -mer probes, targeting

27,958 Entrez genes and 7,419 lincRNAs. Arrays were read

with Agilent Scanner G2565A, using AgilentG3_GX_1Color

as profile. Signals were extracted using FEv.10.7.3.1 and

protocol GE1_107_Sep09 (Agilent Technologies).

\subsection{Microarray Data Analysis}

Microarray intensity data were analyzed by

GeneSpring ${ }^{\mathrm{TM}} \mathrm{GX} 13.1$ software. Data from all samples

were log2-transformed and quantile normalized. Control

probes and probes that were flagged as compromised were

omitted from the analysis. Genes with more than one probe

were averaged if the correlation between probes was $>=0.8$,

while for probes with correlation $<0.8$, the probe with the

highest variance was chosen. Mean intensity values from

each gene from both cell lines, KPL-4 and JIMT1, and from

the three replicate experiments for each cell line ( 6 values in

total per gene) were calculated to increase statistical power.

Statistical analyses were performed on normalized intensity

values using an unpaired Student t-test. Probes with false

discovery rate $\mathrm{p}$-values (Benjamini and Hochberg) less than

0.1 were considered to be significant. mRNA expression data

have been submitted to the Gene Expression Omnibus
(GEO) database [GEO: GSE108324].

\section{2. miR-342-5p Target Predictions}

TargetScan Human version 7.0 [11-13] was employed to predict target genes for miR-342-5p. This database uses an algorithm based on conservation criteria, and was ranked as the most robust compared to several other databases in a previous study [14]. Predictions were filtered for transcripts with a context score threshold of $\leq-0.2$, where the targets with lowest context scores represent the most confident targets. To improve the reliability of the target genes predicted, a second target prediction database, DIANA-microT-CDS $[15,16]$ was also employed. In this algorithm conservation is a feature and not a filter, which increases sensitivity to miRNA targets that are lineage specific. Here, low confidence targets were eliminated by filtering out targets with a total context score threshold of $\geq 0.7$. To identify potential targets for miR-342-5p among the significantly up- and downregulated genes in miR-342-5p transfected versus negative control transfected cell lines, target genes with the indicated thresholds were retrieved and matched to the gene list.

\subsection{Ingenuity Pathway Analysis}

Functional annotation and pathway analysis was performed using Ingenuity Pathway Analysis software (IPA, Ingenuity Systems, Redwood City, CA) on significantly regulated

genes between the cells transfected with miR-342-5p and negative control miRNA. Both up- and down-regulated genes with FDR $<0.1$ after miR-342-5p overexpression, compared to negative control cells, were used as input for the IPA analysis. IPA uses Fisher's exact test to determine whether a canonical pathway is enriched within the genes of interest.

\subsection{Breast Cancer Patients}

Three clinical cohorts with matched mRNA and miRNA expression data from breast cancer patients were employed in this study. From a total of 1283 patients in the METABRIC cohort, 1122 were HER2 negative and 161 were HER2 positive. HER2 status was determined by immunohistochemistry (IHC) and fluorescence in situ hybridization 
(FISH), where tumors were scored HER2 positive if at least

$10 \%$ showed membranous staining and/or if HER2 FISH

was positive.

From the Oslo2 cohort, 363 patients were analyzed, with

301 being HER2 negative and 41 HER2 positive [17]. Amplification

of the human epidermal growth factor receptor 2

(HER2) gene was assessed by a combination of IHC and

chromogenic in situ hybridization (CISH) following standard

guidelines.

Within the 96 primary breast carcinoma samples from the

MicMa cohort, 73 tumors were HER2 negative and 17 HER2

positive. In this cohort HER2 status was

determined by immunohistochemistry

(IHC) and fluorescence in situ hybridization

(FISH), where tumors were scored HER2 positive if at

least $10 \%$ showed membranous staining and/or if HER2

FISH.

Detailed information about tissue collection and patient

information for the METABRIC, Oslo2 and MicMa cohorts

can be found in [17-19] respectively. The Regional Committee

for Medical and Health Research Ethics for southeast Norway

have approved the study (approval number 1.2007.1125.)

The Oslo2 trial does not have substantial long term follow-

up data yet, so survival analyses could not be performed

in this cohort.

\section{RESULTS}

\subsection{Identification of Differentially Regulated Genes after} miR-342-5p Overexpression

To investigate the mechanisms behind the inhibitory effect of miR-342-5p on HER2 signaling and cell growth,

gene expression microarrays were performed after miR-342-

$5 p$ overexpression in two HER2 amplified breast cancer cell

lines, JIMT-1 and KPL-4. After merging probes from the

two cell lines, a total of 581 genes were significantly differentially

expressed between miR-342-5p transfected and negative

control miRNA transfected cells (FDR<0.1) (Suppl.

Table S1). Of these, 383 genes were downregulated and 198 genes were upregulated.

Of the deregulated genes, 139 were predicted targets of miR-342-5p; 128 by TargetScan and 59 by DIANA microTCDS. A total of 48 of the predicted targets were overlapping between the two algorithms (Suppl. Table S1).

\section{2. miR-342-5p can Suppress mRNAs Involved in HER2}

Downstream Signaling Pathways and Cell Junctions

Next, we performed pathway enrichment analyses with the Ingenuity Pathway Analysis (IPA) to examine the biological pathways and processes regulated by miR-342-5p. Pathway analysis of the 581 miR-342-5p-regulated genes identified ERK/MAPK signaling and SAPK/JNK signaling among the five most significantly regulated pathways (Table $\mathbf{1}$ ). Both pathways were estimated to be downregulated after miR-342-5p overexpression, although the ERK/MAPK pathway to a lower extent.

When the pathway analysis was limited to the genes predicted to be miR-342-5p targets, germ cell-sertoli cell junction signaling, sertoli cell-sertoli cell junction signaling and integrin signaling were included among the five most significantly affected pathways after miR-342-5p overexpression (Table 2). Although only integrin-signaling was predicted to be downregulated after miR-342-5p overexpression, the results imply a change in cell-cell connection as all these three pathways provide contact between cells or between cells and extracellular matrix (ECM). In line with this observation, four of the ten most upregulated genes from the total gene list of 581 genes were also associated with cellular contact (Table 3): The guanine exchange factor Rap Guanine Nucleotide Exchange Factor 6 (RAPGEF6), Keratin 80 (KRT80), Claudin 19 (CLDN19) and Delta Like Canonical Notch Ligand 1 (DLL1).

\section{3. $m i R-342-5 p$ can Suppress mRNAs Involved in Mitochondrial}

\section{Dysfunction and Protection of Oxidative Stress}

Deregulation of mitochondrial function was enriched among the miR-342-5p regulated genes, within the top toxicological functions (Table 4). This was also true for enrichment analysis performed on the gene list with only predicted 
targets (Table 2 and 5).

Of the predicted and significantly down-regulated target genes, NADH-cytochrome b5 reductase 3 (CYB5R3), Glutathione peroxidase 4 (GPX4), cytochrome c oxidase (COX) assembly homolog 10 (COX10) and Caspase 9 (CASP9) were associated with mitochondrial dysfunction. Additional interesting mitochondria-associated genes known to have key functions in maintaining mitochondrial integrity and protection of cells against oxidative stress, and which were significantly downregulated upon miR-342-5p overexpression, included methionine sulfoxide reductase B2 (MSRB2), cytochrome c-1 (CYC1) and translocase of inner mitochondrial membrane 17 homolog B (TIMM17B) (Suppl. Table S1). COX10 and CYC1 encode important players in the mitochondrial respiratory chain, and their loss is associated with various mitochondrial complex deficiencies. CYB5R3, GPX4 and MSRB2 have been characterized as key effectors in the protection of oxidative stress [20-22], while TIMM17B is essential for constitutive mitochondrial functions, such as maintenance of electron transport chain activity [23]. Interestingly, except for MSRB2 and CYC1, all these genes were identified as predicted targets in either the TargetScan and/or DIANA algorithms. Furthermore, TIMM17B,

CYB5R3 and GPX4 were also among the ten most downregulated genes after miR-342-5p overexpression (Table 3 ), indicating a strong miR-342-5p induced downregulation of important genes maintaining mitochondrial stability and function.

\section{4. miR-342-5p Expression Correlates with CYB5R3 and}

\section{RAPGEF6 Expression in Breast Cancer Patients}

To investigate correlation between miR-342-5p and its potential target genes, three clinical datasets, METABRIC ( $\mathrm{n}$ $=1283)$, OSLO2 $(n=363)$ and MicMa $(n=99)$, with both mRNA and miRNA expression data, were employed. Among the twenty most down-regulated genes, CYB5R3 was found to significantly negatively correlate with miR-342-5p ( $r<-$ 0.3, p < 0.001) (Suppl. Table S2 and Fig. 1A and B) in the two former datasets. This correlation was weaker in the MicMa cohort (Fig. 1C). Furthermore, the negative correlation was seen within both HER2 positive and HER2 negative patients within the two former dataset, but only in the HER2 negative patients from the MicMa cohort. From the twenty most upregulated genes, RAPGEF6 significantly positively correlated with miR-342-5p $(r>0.3, p<$ 0.01) in the METABRIC dataset, regardless of HER2 status (Suppl. Table S3 and Fig. 2A). In the Oslo2 dataset, RAPGEF6 significantly positively correlated with miR-342$5 p(r>0.3, p=0.01)$ in HER2 positive patients, whereas, the correlation in the HER2 negative subset of patients expression was significant, but modest (Fig. 2B, r=0.22, p < 0.001). In contrast, the correlation between miR-342-5p and RAPGEF6 was only significant within the HER2 negative subset of patients from the MicMa cohort (Fig. 2C). Further analysis within the PAM50 subgroups identified a significant negative correlation between miR-342-5p and CYB5R3 expression within all five subgroups in the METABRIC cohort (Fig. 3A). On the other hand, a positive correlation between miR-342-5p and RAPGEF6 expression was only seen within the Basal, Her2 and Normal-like subgroups (Fig. 3B). In the MicMa cohort, a significant correlation between miR-342-5p expression and CYB5R3 was only seen in the Luminal A subtype (Fig. 4A), while the correlation between miR-342-5p and RAPGEF6 was only present in the Basal subtype (Fig. 4B). Importantly, due to the low number of patients within each subtype of the MicMa cohort, the results from this dataset should be verified in another clinical cohort.

\subsection{Genes with High Correlation to miR-342-5p Expression}

\section{Correlate with Survival}

To test whether the highly correlating genes could be linked to survival, all patients within the METABRIC and MicMa cohorts were divided into two groups based on their CYB5R3 or RAPGEF6 expression; a "low" group denoting samples with CYB5R3 or RAPGEF6 expression below the median, and a "high"' group containing samples with expression levels above the median. As illustrated in Fig. (5A and B), Kaplan-Meier survival curves from the METABRIC 
cohort showed a significant increase in 5- and 10-year overall survival for patients with low CYB5R3 expression, compared to patients with high CYB5R3 expression $(p=0.009$ and $p=0.02$, respectively). The same trend was also seen for patients in the Micma cohort, but was only significantly different at 10 year overall survival (Suppl. Fig. 1A and B). In accordance with the positive correlation of RAPGEF6 and miR-342-5p expression, a significant increase in 5- and 10-year overall survival was seen for patients with high RAPGEF6 expression in the METABRIC cohort $(p=0.007$ and $p=0.027$, respectively, Fig. 5C and D). Although the same trend was seen for RAPGEF6 expression in the MicMa cohort, these results were not significant (Suppl. Fig. 1C and D). For CYB5R3, $\mathrm{n}=641$ for "high" group and $=640$ for "low" group. For RAPGEF6, $\mathrm{n}=641$ for both "high" and "low" groups. P-values were obtained using a log-rank test. Importantly, neither of the two genes showed a significant association with survival when employing univariate cox regression on continuous gene expression (data not shown).

The four different combinations of high/low CYB5R3 and RAPGEF6 expression in combination was also tested, and as illustrated in Fig. (7), the combination of high CYB5R3 and low RAPGEF6 resulted in worse outcome compared to the 3 other combinations in both clinical cohorts. This combination was significantly associated with survival at 5 year overall survival in the METABRIC cohort $(p=0.02$, Fig.

7A), and 10 year overall survival in the MicMa cohort ( $p=$ 0.02, Fig. 7D).

CYB5R3 and RAPGEF6 expression and their correlation to survival were also analyzed within PAM50 subgroups in the METABRIC cohort. For CYB5R3, no correlation to 5 and 10 year survival was seen within any of the subgroups (data not shown). For RAPGEF6, a significantly better survival was found within the Normal-like subgroup at 10 year ( $p=0.016$, data not shown), but not for any of the other subgroups.

\section{DISCUSSION}

The tumors overexpressing HER2 are known to be of high histological grade, with an aggressive clinical behavior $[7,24]$. Targeted therapy against the HER2 receptor has significantly increased progression free and overall survival in these patients [25]. As seen in the Cleopatra study, addition of pertuzumab to trastuzumab and docetaxel, as compared to the addition of placebo, significantly improved overall survival with 15.7 months [26]. However, drug resistance remains a critical issue, and new HER2 targeted therapies are therefore continuously being developed and tested in clinical trials. We previously reported miR-342-5p as a promising candidate with a potential inhibitory effect on HER2 driven breast cancers [9]. We demonstrated that high expression of miR-342-5p was associated with better overall survival and increased time to local recurrence in breast cancer patients. A significant association of high miR-342-5p expression and better survival was also found across all patients in two cohorts of breast cancer patients.

In the present study, the inhibitory and targeting functions of this miRNA were further investigated by analyzing gene expression after overexpressing miR-342-5p in two HER2 positive breast cancer cell lines. Activation of the HER2 receptor leads to cytoplasmic signaling through survival pathways like PI3K/Akt and the MAPK pathways ERK, p38 and JNKs [27]. We previously described the HER2 downstream inhibiting effect of miR-342-5p on the PI3K/AKt and the MAPK/ERK pathways [9]. In the present study, the c-Jun NH2-terminal kinase/stress-activated protein kinase (SAPK/JNK) was identified as one of the most significantly downregulated pathways after miR-342-5p overexpression. Mitogen-Activated Protein Kinase 11 and 13 (MAP3K11 and MAP3K13), which were found downregulated after miR342-5p overexpression, function as positive regulators of the SAPK/JNK signaling pathway through activation of MAPK8/JNK (JNK1). The role of JNK in the HER2 signaling pathway has been subject for recent attention as activation of JNK1 has been shown to promote cell survival in 
HER2-positive breast cancer [28], as well as to modulate acquired resistance to EGFR/HER2-targeted therapies [29-

32]. The identified downregulation of genes involved in the activation of JNK signaling after miR-342-5p overexpression may therefore be, at least partly, responsible for the decrease of the growth of HER2 positive breast cancer cell lines.

The JNKs are activated in response to a variety of cellular stresses, including UV, methylating agents, heat shock and oxidative stress [33], and play an important role in modulating the functions of pro- and anti-apoptotic proteins located in mitochondria [34]. The downregulation of the SAPK/JNK pathway therefore correlates to the effect elicited by miR-342-5p overexpression on mitochondria, which was reflected through the identification of changes in several mitochondria associated pathways upon miR-342-5p overexpression. A potential link between targeting the HER2 pathway and mitochondrial effects correlates with the findings in a study by Grazette et al. [35], where antibody-targeting of HER2 caused a loss of mitochondrial membrane potential and an increase in cell death. Furthermore, blockade of the HER2 receptor has been shown to induce cardiomyocyte death through a mitochondrial pathway that is dependent on ROS production [36]. We identified CYB5R3, GPX4 and MSRB2 among the genes mapped to mitochondrialassociated pathways, which are all known as key effectors in the protection of oxidative stress [20-22, 37-39]. Furthermore, TIMM17B is essential for constitutive mitochondrial functions, and its downregulation has been shown to significantly decrease cell viability [23]. The additional finding of TIMM17B, CYB5R3 and GPX4 among the ten most downregulated genes, as well as being predicted targets for miR-

$342-5 p$, suggests that there may be a potential direct or indirect targeting of either one, or some, of these genes by miR342-5p. Altogether, the results indicate that miR-342-5p overexpression leads to reduced $M A P K / J N K$ signaling and mitochondrial instability, which renders cells more vulnerable to stress and thereby more prone to apoptosis. However, as the role of JNK in apoptosis can be both pro- or antiapoptotic, depending on cell type, nature of the death stimulus, duration of its activation as well as other signaling pathways [40], the effects of the reduced SAPK/JNK signaling upon miR-342-5p overexpression is hard to conclude. CYB5R3, a key effector in the protection of oxidative stress, may represent a potential link between miR-342-5p overexpression and the decrease in HER2 positive breast cancer cell growth. CYB5R3 was identified as a predicted miR-342-5p target within both target prediction algorithms employed. This was also the only gene among the most downregulated genes after miR-342-5p overexpression, which was found anti-correlated to miR-342-5p expression in the three clinical datasets employed. However, when analyzing correlations within the PAM50 subgroups, none of the subgroups showed a consistent link between miR-342-5p and CYB5R3 expression across the clinical cohorts. This was also true for the survival analysis, where a significantly better outcome of low $C Y B 5 R 3$, either separately or in combination with high RAPGEF6 expression, was only seen within all patients combined. The lack of correlation within the HER2 positive subset could be explained by the previous identified low levels of miR-342-5p expression within HER2 positive breast cancer patients [9]. There were also fewer HER2 positive patients in all clinical cohorts employed.

Loss of cell polarity and adhesion is a hallmark of many epithelial cancers. In relation to this, we found that within the gene list containing only miR-342-5p predicted targets, three of the five most significantly deregulated pathways reflected cell junction or integrin signaling. Among the twenty most upregulated genes after miR-342-5p overexpression in the HER2 positive cell lines, only RAPGEF6 was found to positively correlate with miR-342-5p expression in the METABRIC cohort. This was also true for the Oslo2 cohort, but only in the HER2 negative breast cancer patients. A significant association with survival was also found for RAPGEF6 in the METABRIC cohort, where higher expression was correlated to increased survival. RAPGEF6, also known as $P D Z-G E F 2$, is a guanine exchange factor, and has 
been shown to play a role in adherens junctions between cells, integrin junctions to the matrix, actin organization and migration in epithelial and lymphatic cells [41, 42]. RAPGEF6 itself was not a predicted target for miR-342-5p, and its upregulation after miR-342-5p overexpression may therefore be the result of an indirect mechanism rather than a direct targeting. In patients from the METABRIC cohort, RAPGEF6 was significantly positively correlated to miR342-5p expression within the Basal, HER2 and Normal-like PAM50 subgroups. A trend towards better survival for patients with high levels of RAPGEF6 within the Normal-like subgroup was seen at 5 years $(p=0.09$, data not shown), although it only reached statistical significance at 10 years ( $p$ $=0.016$, data not shown). A significantly positive correlation between miR-342-5p and RAPGEF6 was also seen within the Oslo2 and MicMa datasets, however, no consistency could be found regarding subgroup specificity.

\section{CONCLUSION}

To conclude, the results presented provide mechanistic insight for the role of miR-342-5p in inhibiting the growth of HER2 overexpressing breast cancers. Several of the findings suggest that the observed decrease in HER2 driven tumor growth is, at least in part, the result of a miR-342-5p-induced downregulation of HER2 downstream signaling pathways, as well as a downregulation of important protectors against oxidative stress. Here, $C Y B 5 R 3$ may represent an important contributor to the observed decrease in cell growth. However, as the present study is limited to the use of only HER2 positive cell line analyses, we cannot conclude whether the hypothesized targeting of CYB5R3 by miR-342-5p is HER2 specific. Additional studies in HER2 negative cell lines are therefore warranted. Importantly, miR-342 has previously been shown to have significantly lower expression in TP53 mutated tumors [19], and as being tightly correlated to estrogen receptor (ER) and immune cell expression [43]. As these parameters are very likely to impact on survival, it could also explain why better survival is only seen across all patients and not within the PAM50 subgroups. Nevertheless, the results underscore a targeting effect from miR-342-5p on the mitochondrial transmembrane potential and respiratory chain complexes, and a reduction in the defense mechanisms against oxidative stress. As direct targeting of the HER2 receptor is frequently found to be insufficient to inhibit the growth of HER2 positive breast cancers, as well as the development of resistance to such therapies, miRNA-mediated targeting could accomplish a wider effect and thereby offer a promising new therapeutic intervention for these patients.

\section{ETHICS APPROVAL AND CONSENT TO PARTICIPATE}

Not applicable.

\section{HUMAN AND ANIMAL RIGHTS}

No Animals/Humans were used for studies that are the basis of this research.

\section{CONSENT FOR PUBLICATION}

Not applicable.

\section{CONFLICT OF INTEREST}

The authors declare no conflict of interest, financial or otherwise.

\section{ACKNOWLEDGEMENTS}

We would like to acknowledge Oslo Breast Cancer Research Consortium (OSBREAC).

This research was supported by funds from the Norwegian Cancer Society (\# 419628 138296).

\section{SUPPLEMENTARY MATERIAL}

Supplementary material is available on the publisher's website along with the published article.

\section{REFERENCES}

[1] Pasquinelli AE. MicroRNAs and their targets: recognition, regulation and an emerging reciprocal relationship. Nat Rev Genet. 
2012 Apr; 13(4): 271-82. PubMed PMID: 22411466. Epub 2012/03/14. eng.

[2] Friedman JM, Jones PA. MicroRNAs: critical mediators of differentiation, development and disease. Swiss Med Wkly. 2009 Aug 22; 139(33-34): 466-72. PubMed PMID: 19705306. Pubmed Central PMCID: 2854010. Epub 2009/08/26. eng.

[3] Garzon R, Calin GA, Croce CM. MicroRNAs in Cancer. Annu Rev Med. 2009; 60: 167-79. PubMed PMID: 19630570. Epub 2009/07/28. eng.

[4] Iuliano R, Vismara MF, Dattilo V, Trapasso F, Baudi F, Perrotti N. The role of microRNAs in cancer susceptibility. Biomed Res Int. 2013; 2013: 591931. PubMed PMID: 23586049. Pubmed Central PMCID: 3615597. Epub 2013/04/16. eng.

[5] Selbach M, Schwanhausser B, Thierfelder N, Fang Z, Khanin R, Rajewsky N. Widespread changes in protein synthesis induced by microRNAs. Nature. 2008 Sep 4; 455(7209): 58-63. PubMed PMID: 18668040. Epub 2008/08/01. eng.

[6] Garzon R, Marcucci G, Croce CM. Targeting microRNAs in cancer: rationale, strategies and challenges. Nat Rev Drug Discov. 2010 Oct; 9(10): 775-89. PubMed PMID: 20885409. Pubmed Central PMCID: 3904431. Epub 2010/10/05. eng. [7] Slamon DJ, Clark GM, Wong SG, Levin WJ, Ullrich A, McGuire WL. Human breast cancer: correlation of relapse and survival with amplification of the HER-2/neu oncogene. Science. 1987 Jan 9; 235(4785): 177-82. PubMed PMID: 3798106. Epub 1987/01/09. eng.

[8] Gonzalez-Angulo AM, Litton JK, Broglio KR, Meric-Bernstam F, Rakkhit R, Cardoso F, et al. High risk of recurrence for patients with breast cancer who have human epidermal growth factor receptor 2-positive, node-negative tumors $1 \mathrm{~cm}$ or smaller. J Clin Oncol. 2009 Dec 1; 27(34): 5700-6. PubMed PMID: 19884543. Pubmed Central PMCID: 2792998. Epub 2009/11/04. eng. [9] Leivonen SK, Sahlberg KK, Makela R, Due EU, Kallioniemi O, Borresen-Dale AL, et al. High-throughput screens identify microRNAs essential for HER2 positive breast cancer cell growth. Mol Oncol. 2014 Feb; 8(1): 93-104. PubMed PMID: 24148764. Epub 2013/10/24. eng.

[10] Kurebayashi J, Otsuki T, Tang CK, Kurosumi M, Yamamoto S,
Tanaka $\mathrm{K}$, et al. Isolation and characterization of a new human breast cancer cell line, KPL-4, expressing the Erb B family receptors and interleukin-6. Br J Cancer. 1999 Feb; 79(5-6): 70717. PubMed PMID: 10070858. Pubmed Central PMCID: 2362677. [11] Lewis BP, Burge CB, Bartel DP. Conserved seed pairing, often flanked by adenosines, indicates that thousands of human genes are microRNA targets. Cell. 2005 Jan 14; 120(1): 15-20. PubMed PMID: 15652477. Epub 2005/01/18. eng.

[12] Grimson A, Farh KK, Johnston WK, Garrett-Engele P, Lim LP, Bartel DP. MicroRNA targeting specificity in mammals: determinants beyond seed pairing. Mol Cell. 2007 Jul 6; 27(1): 91105. PubMed PMID: 17612493. Pubmed Central PMCID: 3800283. Epub 2007/07/07. eng.

[13] Friedman RC, Farh KK, Burge CB, Bartel DP. Most mammalian mRNAs are conserved targets of microRNAs. Genome Res. 2009 Jan; 19(1): 92-105. PubMed PMID: 18955434. Pubmed Central PMCID: 2612969. Epub 2008/10/29. eng.

[14] Baek D, Villen J, Shin C, Camargo FD, Gygi SP, Bartel DP. The impact of microRNAs on protein output. Nature. 2008 Sep 4; 455(7209): 64-71. PubMed PMID: 18668037. Pubmed Central PMCID: 2745094. Epub 2008/08/01. eng.

[15] Maragkakis M, Reczko M, Simossis VA, Alexiou P, Papadopoulos GL, Dalamagas T, et al. DIANA-microT web server: elucidating microRNA functions through target prediction. Nucleic acids research. 2009 Jul; 37(Web Server issue): W273-6. PubMed PMID: 19406924. Pubmed Central PMCID: 2703977.

[16] Paraskevopoulou MD, Georgakilas G, Kostoulas N, Vlachos IS, Vergoulis T, Reczko M, et al. DIANA-microT web server v5.0: service integration into miRNA functional analysis workflows. Nucleic acids research. 2013 Jul; 41(Web Server issue): W169-73. PubMed PMID: 23680784. Pubmed Central PMCID: 3692048. Epub 2013/05/18. eng. [17] Aure MR, Vitelli V, Jernstrom S, Kumar S, Krohn M, Due EU, et al. Integrative clustering reveals a novel split in the luminal A subtype of breast cancer with impact on outcome. Breast Cancer Research. 2017 Mar 29; 19. PubMed PMID: WOS: 000397653500001. English.

[18] Curtis C, Shah SP, Chin SF, Turashvili G, Rueda OM, Dunning 
MJ, et al. The genomic and transcriptomic architecture of 2,000 breast tumours reveals novel subgroups. Nature. 2012 Jun 21; 486(7403): 346-52. PubMed PMID: WOS: 000305466800033. English.

[19] Enerly E, Steinfeld I, Kleivi K, Leivonen SK, Aure MR, Russnes HG, et al. miRNA-mRNA integrated analysis reveals roles for miRNAs in primary breast tumors. PloS one. 2011 Feb 22; 6(2): e16915. PubMed PMID: 21364938. Pubmed Central PMCID: 3043070.

[20] Siendones E, SantaCruz-Calvo S, Martin-Montalvo A, Cascajo MV, Ariza J, Lopez-Lluch G, et al. Membrane-bound CYB5R3 is a common effector of nutritional and oxidative stress response through FOXO3a and Nrf2. Antioxid Redox Signal. 2014 Oct 20; 21(12): 1708-25. PubMed PMID: 24450884. Pubmed Central PMCID: 4186635. Epub 2014/01/24. eng.

[21] Ran Q, Liang H, Gu M, Qi W, Walter CA, Roberts LJ, 2nd, et al. Transgenic mice overexpressing glutathione peroxidase 4 are protected against oxidative stress-induced apoptosis. J Biol Chem. 2004 Dec 31; 279(53): 55137-46. PubMed PMID: 15496407. Epub 2004/10/22. eng.

[22] Cabreiro F, Picot CR, Perichon M, Castel J, Friguet B, Petropoulos I. Overexpression of mitochondrial methionine sulfoxide reductase B2 protects leukemia cells from oxidative stress-induced cell death and protein damage. J Biol Chem. 2008 Jun 13; 283(24): 16673-81. PubMed PMID: 18424444.

[23] Sinha D, Srivastava S, Krishna L, D'Silva P. Unraveling the intricate organization of mammalian mitochondrial presequence translocases: existence of multiple translocases for maintenance of mitochondrial function. Mol Cell Biol. 2014 May; 34(10): 1757-75. PubMed PMID: 24636990. Pubmed Central PMCID: 4019027. Epub 2014/03/19. eng.

[24] Slamon DJ, Godolphin W, Jones LA, Holt JA, Wong SG, Keith DE, et al. Studies of the HER-2/neu proto-oncogene in human breast and ovarian cancer. Science. 1989 May 12; 244(4905): 707-

\section{PubMed PMID: 2470152.}

[25] Dawood S, Broglio K, Buzdar AU, Hortobagyi GN, Giordano SH. Prognosis of women with metastatic breast cancer by HER2 status and trastuzumab treatment: an institutional-based review. J Clin
Oncol. 2010 Jan 1; 28(1): 92-8. PubMed PMID: 19933921.

Pubmed Central PMCID: 2799236. Epub 2009/11/26. eng.

[26] Swain SM, Baselga J, Kim SB, Ro J, Semiglazov V, Campone M, et al. Pertuzumab, Trastuzumab, and Docetaxel in HER2-Positive Metastatic Breast Cancer. New England Journal of Medicine. 2015 Feb 19; 372(8): 724-34. PubMed PMID: WOS: 000349818700006. English.

[27] Yarden Y, Sliwkowski MX. Untangling the ErbB signalling network. Nat Rev Mol Cell Biol. 2001 Feb; 2(2): 127-37. PubMed PMID: 11252954.

[28] Han JS, Crowe DL. Jun amino-terminal kinase 1 activation promotes cell survival in ErbB2-positive breast cancer. Anticancer Res. 2010 Sep; 30(9): 3407-12. PubMed PMID: 20944115.

[29] Manole S, Richards EJ, Meyer AS. JNK Pathway Activation Modulates Acquired Resistance to EGFR/HER2-Targeted Therapies. Cancer Res. 2016 Sep 15; 76(18): 5219-28. PubMed PMID: 27450453. Pubmed Central PMCID: 5026573.

[30] Phelps-Polirer K, Abt MA, Smith D, Yeh ES. Co-Targeting of JNK and HUNK in Resistant HER2-Positive Breast Cancer. PloS one. 2016; 11(4): e0153025. PubMed PMID: 27045589. Pubmed Central PMCID: 4821489.

[31] Phelps-Polirer K, Abt MA, Smith D, Yeh ES. Co-Targeting of JNK and HUNK in Resistant HER2-Positive Breast Cancer. PloS one. 2016 Apr 5; 11(4). PubMed PMID: WOS: 000373599600061. English.

[32] Gschwantler-Kaulich D, Grunt TW, Muhr D, Wagner R, Kolbl H, Singer CF. HER Specific TKIs Exert Their Antineoplastic Effects on Breast Cancer Cell Lines through the Involvement of STAT5 and JNK. PloS one. 2016 Jan 6; 11(1). PubMed PMID: WOS: 000367805100041. English.

[33] Kyriakis JM, Banerjee P, Nikolakaki E, Dai TA, Rubie EA, Ahmad MF, et al. The Stress-Activated Protein-Kinase Subfamily of C-Jun Kinases. Nature. 1994 May 12; 369(6476): 156-60. PubMed PMID: WOS: A1994NK97100052. English.

[34] Kharbanda S, Saxena S, Yoshida K, Pandey P, Kaneki M, Wang $\mathrm{QH}$, et al. Translocation of SAPK/JNK to mitochondria and interaction with $\mathrm{Bcl}-\mathrm{x}(\mathrm{L})$ in response to DNA damage (vol 275, pg 322, 2000). Journal of Biological Chemistry. 2000 Jun 23; 275(25): 
19433-. PubMed PMID: WOS: 000087815900107. English.

[35] Grazette LP, Matsui T, Rosenzweig A. Inhibition of erbB2 causes mitochondrial dysfunction and impaired growth response in cardiomyocytes through altered $\mathrm{Bcl}-\mathrm{xS} / \mathrm{Bcl}-\mathrm{xL}$ signaling: Implications for herceptin-induced cardiomyopathy. Circulation. 2004 Oct 26; 110(17): 8-. PubMed PMID: WOS:

000224783500030. English.

[36] Gordon LI, Burke MA, Singh ATK, Prachand S, Lieberman ED, Sun L, et al. Blockade of the erbB2 Receptor Induces Cardiomyocyte Death through Mitochondrial and Reactive Oxygen Species-dependent Pathways. Journal of Biological Chemistry. 2009 Jan 23; 284(4): 2080-7. PubMed PMID: WOS:

000262545600016. English.

[37] Liang H, Van Remmen H, Frohlich V, Lechleiter J, Richardson A, Ran Q. Gpx4 protects mitochondrial ATP generation against oxidative damage. Biochemical and biophysical research communications. 2007 May 18; 356(4): 893-8. PubMed PMID: 17395155.

[38] Brigelius-Flohe R. Tissue-specific functions of individual glutathione peroxidases. Free Radic Biol Med. 1999 Nov; 27(9-10): 951-65. PubMed PMID: 10569628.

[39] de Cabo R, Siendones E, Minor R, Navas P. CYB5R3: a key player in aerobic metabolism and aging? Aging (Albany NY). 2010; 2(1):

63-8. PubMed PMID: 20228936. Pubmed Central PMCID:

2837205. Epub 2009/01/01. eng

[40] Liu J, Lin AN. Role of JNK activation in apoptosis: A doubleedged sword. Cell Research. 2005 Jan; 15(1): 36-42. PubMed PMID: WOS: 000226960600008 . English.

[41] Dube N, Kooistra MRH, Pannekoek WJ, Vliem MJ, Oorschot V, Klumperman J, et al. The RapGEF PDZ-GEF2 is required for maturation of cell-cell junctions. Cellular Signalling. 2008 Sep; 20(9): 1608-15. PubMed PMID: WOS: 000258368400003 English.

[42] Severson EA, Lee WY, Capaldo CT, Nusrat A, Parkos CA. Junctional adhesion molecule A interacts with Afadin and PDZGEF2 to activate Rap1A, regulate beta1 integrin levels, and enhance cell migration. Mol Biol Cell. 2009 Apr; 20(7): 1916-25.

PubMed PMID: 19176753. Pubmed Central PMCID: 2663925.
[43] Dvinge H, Git A, Graf S, Salmon-Divon M, Curtis C, Sottoriva A, et al. The shaping and functional consequences of the microRNA landscape in breast cancer. Nature. 2013 May 16; 497(7449): 37882. PubMed PMID: WOS: 000318952000040. English. 
Table 1. Top five deregulated predicted canonical pathways after miR-342-5p overexpression.

\begin{tabular}{|c|c|c|c|}
\hline Top Canonical Pathway & p-value & Z-score** & Pathway-related Molecules \\
\hline \hline Geranylgeranyldiphosphate Biosynthesis & 0,00207 & NA & COX10, GGPS1 \\
\hline Sumoylation Pathway & 0,00225 & 1,342 & ARHGDIB, CTBP1, RFC5, SAE1*, SENP2, SUMO1*, UBA2 \\
\hline Role of BRCA1 in DNA Damage Response & 0,00351 & NA & ARID2, ARID1A, E2F8, FANCF, MDC1, RFC5, SMARCA4, \\
\hline SRK/MAPK Signaling & 0,0133 & $-0,333$ & CRKL, DUSP4, GRB2*, MKNK2*, PAK4, PIK3R2, PPP1CA, \\
PPP2R3B, RAC2, SOS2
\end{tabular}

**Z-score of $>1$ predicts activation of the pathway, whereas $z$-scores of $<-1$ predicts inhibition of the pathway. NA indicates that no activity pattern is available. *Gene predicted as target for miR-342-5p through TargetScan and/or DIANA microT-CDS.

Table 2. Top five deregulated predicted canonical pathways after miR-342-5p overexpression, among genes predicted as potential miR-342-5p targets.

\begin{tabular}{|c|c|c|c|}
\hline Top Canonical Pathway & p-value & z-score $* *$ & Pathway-related Molecules \\
\hline Germ Cell-Sertoli Cell junction Signaling & 0,0031 & NA & ACTN4, GRB2, MAP3K11, MAP3K13, RAC2 \\
\hline CD27 Signaling in Lymphocytes & 0,0034 & NA & CASP9, MAP3K11, MAP3K13 \\
\hline Sertoli Cell-Sertoli Cell junction Signaling & 0,0035 & $\mathrm{NA}$ & ACTN4, CLDN7, MAP3K11, MAP3K13 \\
\hline Integrin Signaling & 0,0083 & $-1,342$ & ACTN4, ARF, GRB2, MAP3K11, PFN1, RAC2 \\
\hline Mitochondrial Dysfunction & 0,017 & NA & CASP9, COX10, CYB5R3, GPX4 \\
\hline
\end{tabular}

$* * Z$-score of $>1$ predicts activation of the pathway, whereas $z$-scores of $<-1$ predicts inhibition of the pathway. NA indicates that no activity pattern is available.

Table 3. Top ten fold change regulated molecules after miR-342-5p overexpression.

\begin{tabular}{|c|c|c|c|}
\hline \multicolumn{2}{|c|}{ Downregulated } & \multicolumn{2}{c|}{ Upregulated } \\
\hline Gene Symbol & Expression Log Ratio & Gene Symbol & 2,23 \\
\hline \hline TIMM17B* & $-2,65$ & RAPGEF6 & 2,13 \\
\hline MMP24-AS1 & $-2,55$ & KRT80 & 1,66 \\
\hline CYB5R3* & $-2,45$ & SLC45A4 & 1,37 \\
\hline HS1BP3 & $-2,34$ & SFT2D2 & 1,32 \\
\hline CAPN15 & $-2,30$ & ST3GAL4-AS1 & 1,16 \\
\hline FBRSL1 & $-2,29$ & CLDN19 & 1,11 \\
\hline GPX4* & $-2,19$ & DLL1 & 1,11 \\
\hline ATP5SL* & $-2,19$ & CCDC19 & 1,10 \\
\hline ITFG3* & $-2,15$ & IL33 & \\
\hline SH3GL1* & $-2,13$ & CHPF & \\
\hline
\end{tabular}

*Gene predicted as target for miR-342-5p through TargetScan and/or DIANA microT-CDS. 
Table 4. Top five deregulated toxicity pathways after miR-342-5p transfection.

\begin{tabular}{|c|c|c|}
\hline Top Tox Pathway & p-value & Pathway-related Molecules* \\
\hline \hline Mitochondrial Dysfunction & 0,0493 & $\begin{array}{c}\text { CASP9*, COX10*, CYB5R3*, CYC1, DHODH, } \\
\text { GPX4*, NDUFAB1 }\end{array}$ \\
\hline $\begin{array}{c}\text { Nongenotoxic Hepatocarcinogenicity Biomarker Panel } \\
\text { Ancreases Transmembrane Potential of Mitochondria and Mitochondrial } \\
\text { Membrane }\end{array}$ & 0,0637 \\
\hline Increases Liver Steatosis & 0,0678 & MSRB2, CASP9*, ARID1A, PIM1 \\
\hline Hypoxia-inducible Factor Signaling & 0,10 & MARK2*, TICAM1*, CRTC3*, CNOT3* \\
\hline
\end{tabular}

*Gene predicted as target for miR-342-5p through TargetScan and/or DIANA microT-CDS.

Table 5. Top five deregulated toxicity pathways after miR-342-5p transfection, among genes predicted as potential miR-342-5p targets.

\begin{tabular}{|c|c|c|}
\hline Top Tox Pathway & p-value & Pathway-related Molecules \\
\hline \hline Increases Liver Steatosis & 0,0023 & CNOT3, CRTC3, MARK2, TICAM1 \\
\hline Mitochondrial Dysfunction & 0,0180 & CASP9, COX10, CYB5R3, GPX4 \\
\hline Pro-apoptosis & 0,0240 & CASP9, DIABLO \\
\hline Renal Ischemic Resistance Panel (Rat) & 0,0552 & FOXM1 \\
\hline Biogenesis of Mitochondria & 0,1070 & COX10 \\
\hline
\end{tabular}


A:

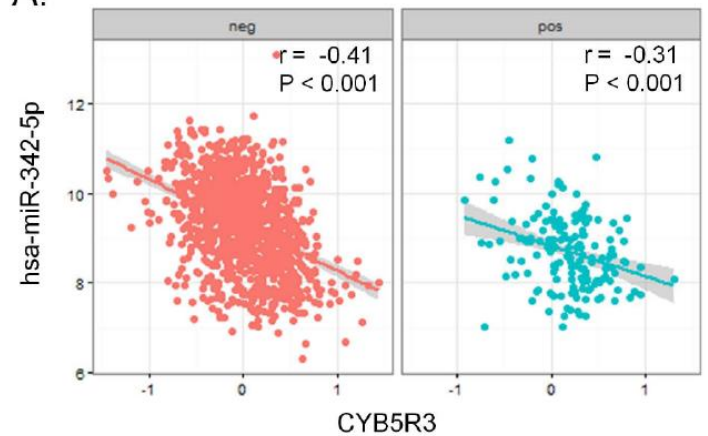

C:

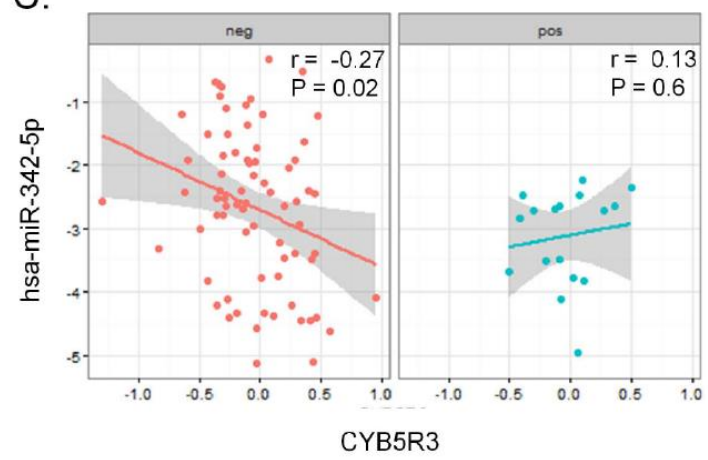

B:

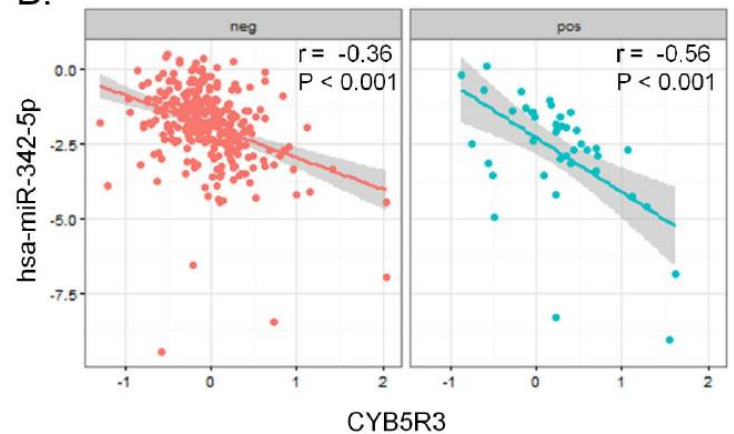

Fig. (1). Correlation between miR-342-5p expression and CYB5R3 mRNA expression in HER2 positive and HER2 negative breast cancer patients in the METABRIC (A), Oslo2 (B) and MicMa (C) cohorts.

A:

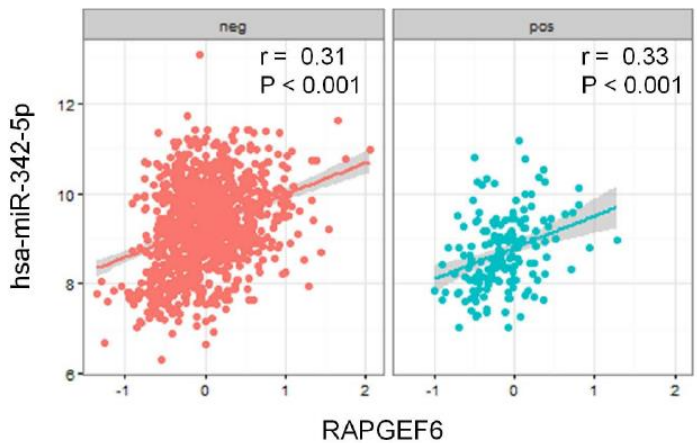

C:

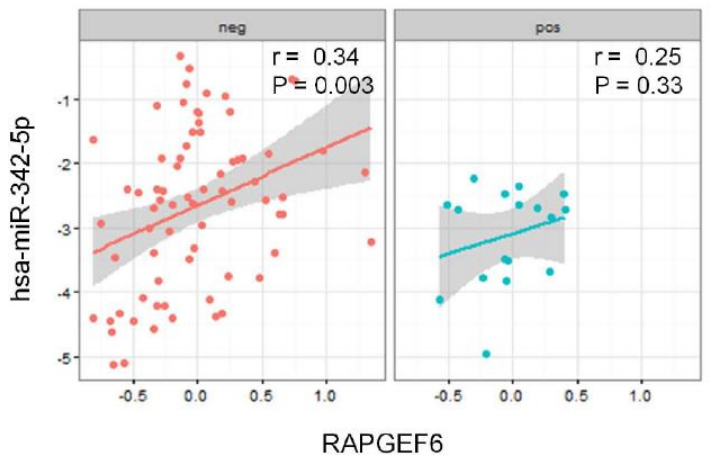

B:

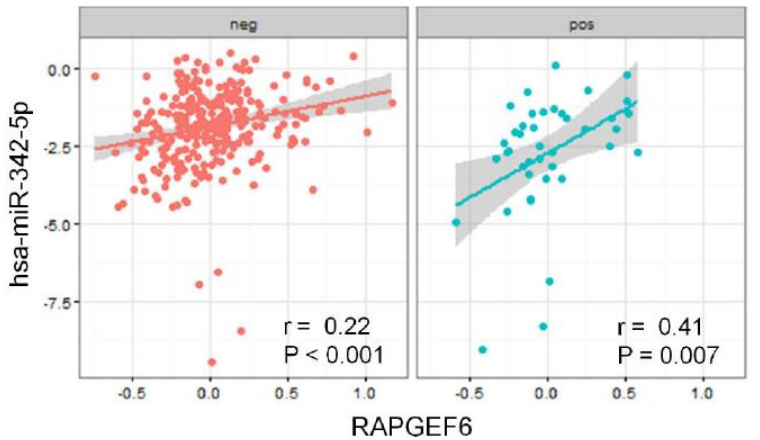

Fig. (2). Correlation between miR-342-5p expression and RAPGEF6 mRNA expression in HER2 positive and HER2 negative breast cancer patients in the METABRIC (A), Oslo2 (B) and MicMa (C) cohorts. 
A:

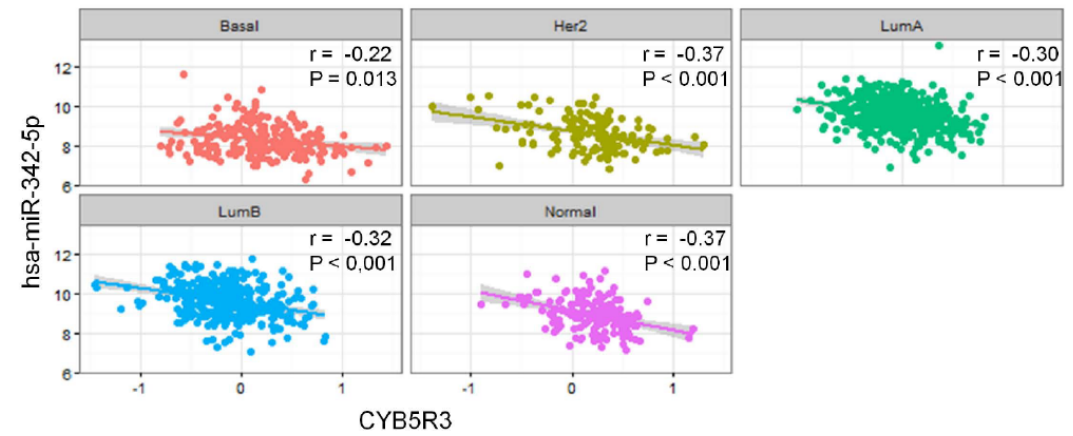

B:

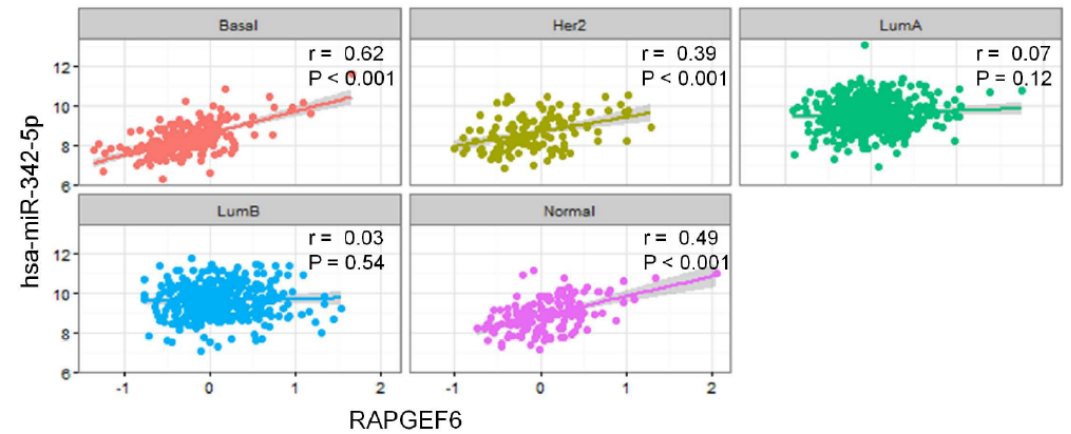

Fig. (3). Correlation between hsa-miR-342-5p expression and CYB5R3 (A) and RAPGEF6 (B) mRNA expression within PAM50 subgroups in the METABRIC cohort.

A:

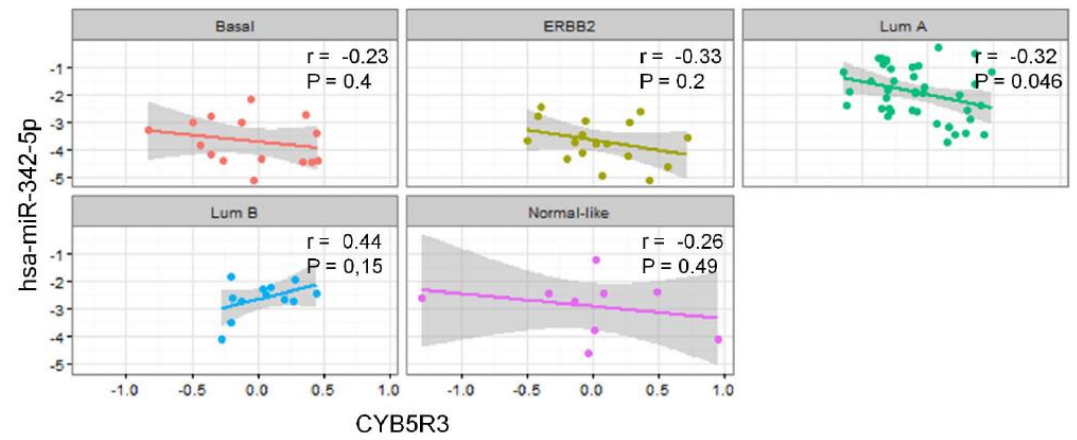

B:

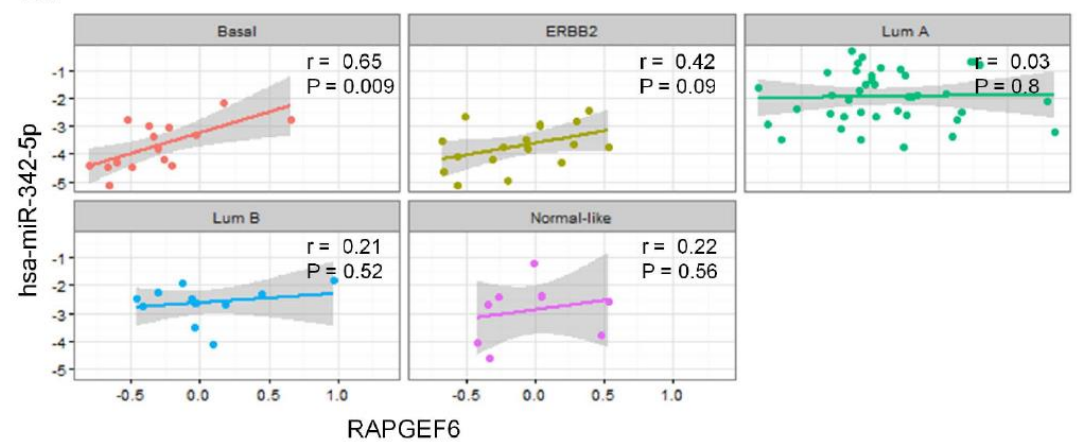

Fig. (4). Correlation between hsa-miR-342-5p expression and CYB5R3 (A) and RAPGEF6 (B) mRNA expression within PAM50 subgroups in the MicMa cohort. 
A:

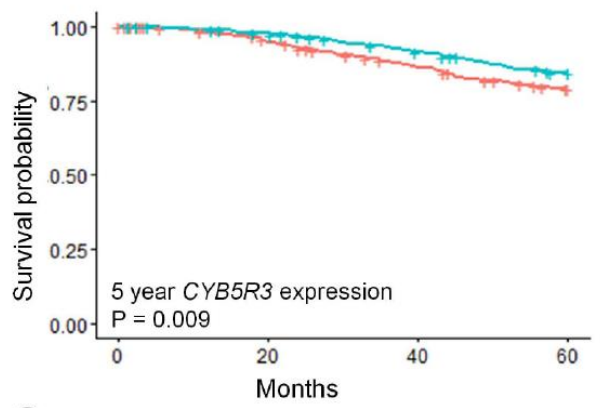

C:

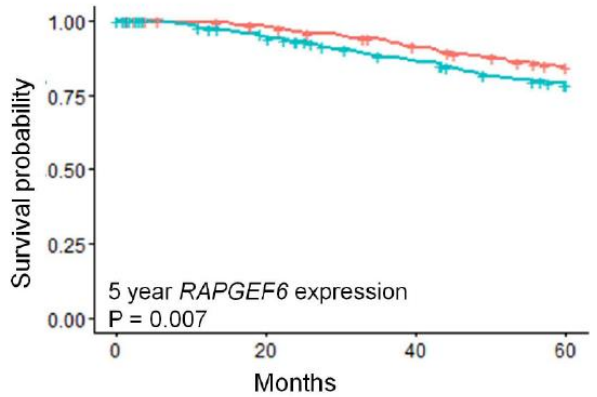

B:

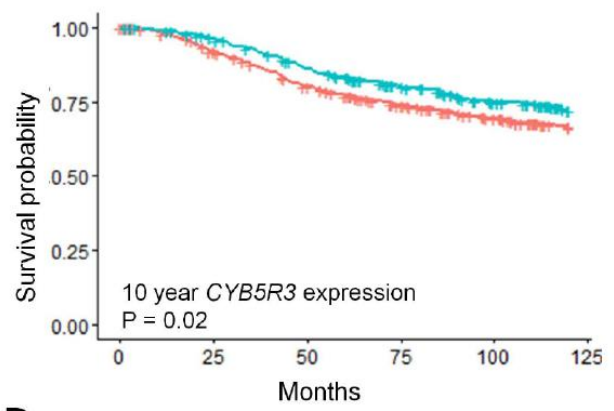

D:

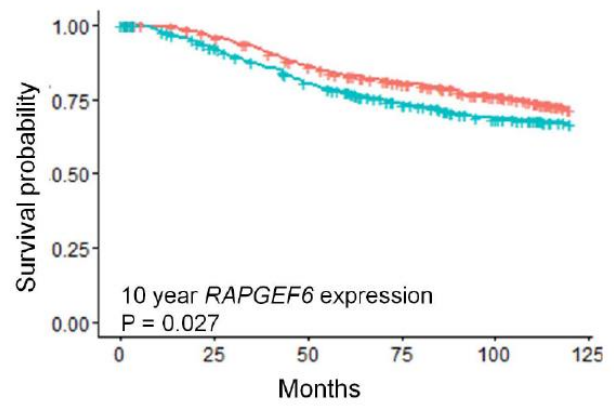

Low gene expression

Fig. (5). Kaplan-Meier curves from the METABRIC cohort showing 5 year (A and C) and 10 year (B and D) overall survival for CYB5R3 (A and $\mathbf{B})$ and RAPGEF6 (C and $\mathbf{D})$ expression. The "low" group denotes samples from breast cancer patients with gene expression below the median and the "high" group contains samples with expression above the median.

A:

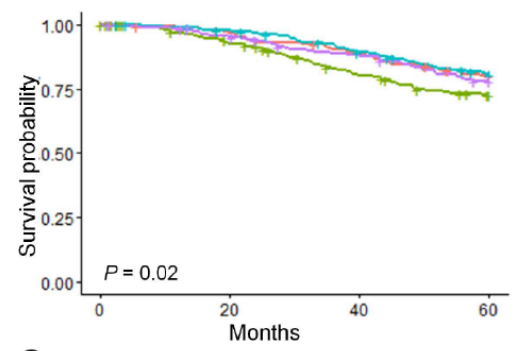

C:

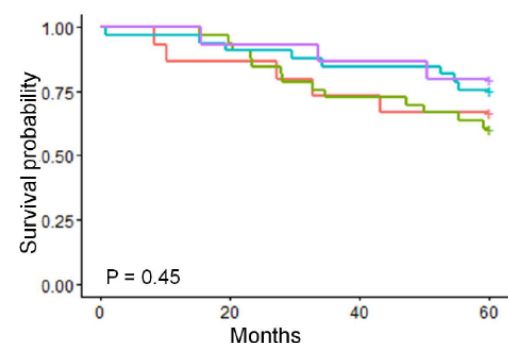

B:

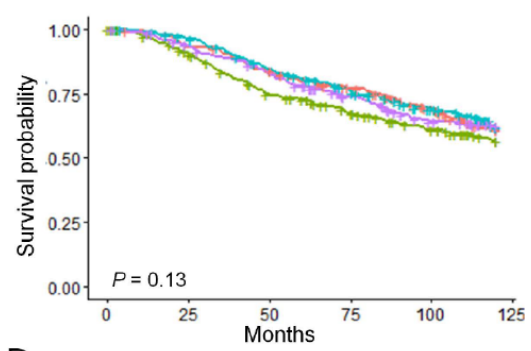

D:

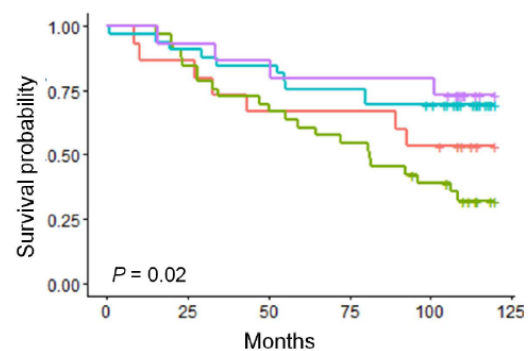

$\begin{array}{llll}- & \text { High CYB5R3 and Low RAPGEF6 } & + & \text { Low CYB5R3 and Low RAPGEF6 } \\ - & \text { High CYB5R3 and High RAPGEF6 } & + & \text { Low CYB5R3 and High RAPGEF6 }\end{array}$

Fig. (6). Kaplan-Meier curves from the METABRIC (A and B) and MicMa (C and D) cohort showing 5 year (A and $\mathbf{C})$ and 10 year (B and D) overall survival for the combined effect of $C Y B 5 R 3$ and RAPGEF6 expression. The "low" group denotes samples from breast cancer patients with gene expression below the median and the "high" group contains samples with expression above the median. P-values were obtained using a log-rank test. 
A:

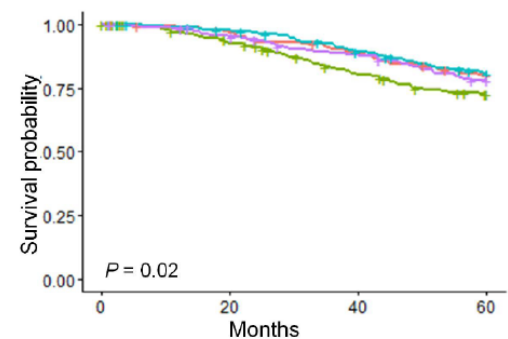

C:

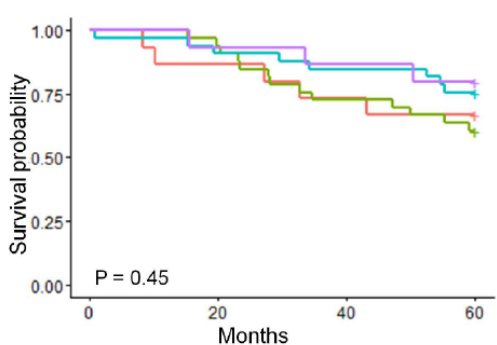

B:

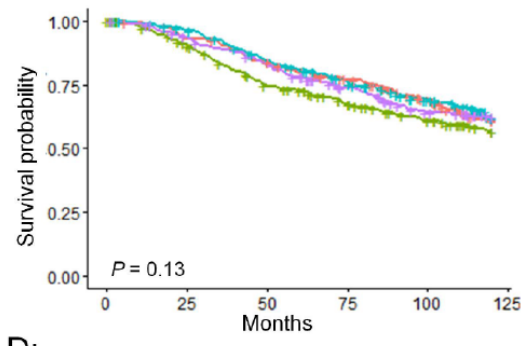

D:

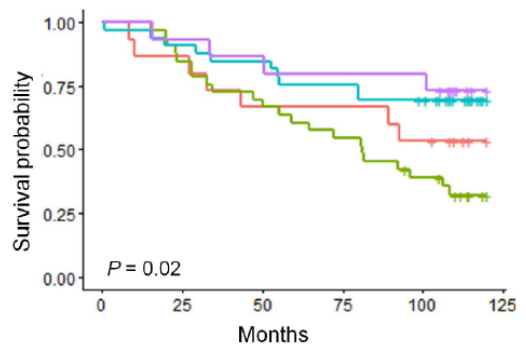

$\mp$ High CYB5R3 and Low RAPGEF

Low CYB5R3 and Low RAPGEF6

High CYB5R3 and High RAPGEF6

Low CYB5R3 and High RAPGEF6

Fig. (7). Kaplan-Meier curves from the METABRIC (A and $\mathbf{B})$ and MicMa $(\mathbf{C}$ and $\mathbf{D})$ cohort showing 5 year $(\mathbf{A}$ and $\mathbf{C})$ and 10 year $(\mathbf{B}$ and D) overall survival for the combined effect of CYB5R3 and RAPGEF6 expression. The "low" group denotes samples from breast cancer patients with gene expression below the median and the "high" group contains samples with expression above the median. P-values were obtained using a log-rank test. 